\title{
Bibliotecarios universitarios - Profesores. ¿Caminos convergentes?
}

\author{
María João Amante*, Ana Extremeño*^
}

Resumen: Los cambios que el proceso de Bolonia ha impuesto en la educación universitaria no sólo atañen a la docencia sino también a organismos incluidos dentro de la propia institución cuya aportación en el proceso enseñanza-aprendizaje es vital para cumplir los requisitos exigidos. Entre ellos, la biblioteca universitaria y sus responsables ocupan un lugar central que los obliga a replantearse sus cometidos profesionales y a adoptar una metodología de trabajo que ayude a la adquisición de las competencias y habilidades que actualmente la sociedad demanda. Un primer paso para delimitar el nuevo rol del bibliotecario universitario consiste en analizar las percepciones que los docentes tienen sobre ellos y, de esta manera, planificar la necesaria e imprescindible colaboración entre ambos colectivos.

Palabras clave: Bibliotecas universitarias, relaciones bibliotecario-profesor, análisis bibliográfico.

\section{University librarians - Teaching staff. Convergent paths?}

Abstract: The changes in higher education brought about by the Bologna process not only affect teaching, but also units within the institution that play a critical role in assuring compliance of the teaching-learning process with the requirements. Among them is the university library, whose officers occupy a key position that requires them to rethink their professional responsibilities and to adopt a methodology for facilitating the acquisition of the competencies and skills demanded by today's society. A first step for defining the new role of university librarians consists of analyzing the faculty's perceptions of them, in order to plan for the necessary collaboration between the two collectives.

Keywords: University library, librarian-teacher relationship, bibliographic analysis.

\section{Introducción}

En la sociedad del conocimiento actual se están produciendo cambios en las instituciones de enseñanza superior que se enfrentan a nuevas necesidades para la realización de sus proyectos docentes los cuales implican nuevos saberes,

* ISCTE - Instituto Universitario de Lisboa, Servicios de Información y Documentación. Portugal. Correo-e: maria.amante@iscte.pt.

** Universidad de Alcalá, Facultad de Documentación. Alcalá de Henares. Madrid. Correo-e: ana.extre@uah.es.

Recibido: 29-03-2011; 2. ${ }^{a}$ versión: 07-06-2011; 3. ${ }^{a}$ versión: 21-07-2011; 4. ${ }^{a}$ versión: 29-09-2011; aceptado: $13-10-2011$. 
competencias y habilidades para poder dar respuestas adecuadas a las exigencias de calidad y a un nuevo modelo pedagógico, derivado del proceso de Bolonia, centrado en el estudiante y en el aprendizaje a lo largo de la vida y que exige una nueva metodología de enseñanza donde, entre otros asuntos, la colaboración entre toda la comunidad universitaria es más importante que nunca. Una primera observación reside en el hecho de que en las universidades existen distintos colectivos que han venido trabajando de forma aislada y que, a partir de ahora, necesitan desarrollar dinámicas de trabajo en red y en equipos multidisciplinares, lo que significa una estructura organizacional distinta y "...una manera diferente de definir los límites en términos de toma de decisiones en nuestras instituciones.» (Hawkins y Battin, 1997).

Este trabajo se dirige a dos colectivos, profesores y bibliotecarios universitarios, que forzosamente han de caminar juntos, puesto que tanto unos como otros tienen objetivos comunes: involucrar a los estudiantes en el pensamiento crítico, así como en el aprendizaje orientado a una disciplina que depende de la organización del conocimiento y la comprensión de los discursos disciplinarios e interdisciplinarios (Hutchins, 2005). Para lograr esos objetivos la universidad debe constituir un entorno favorable a la consideración del estatus de cada uno de ellos y a los acuerdos de colaboración entre ambos.

Son, precisamente, ese estatus y esa necesidad de acuerdos lo que, en numerosas ocasiones y durante mucho tiempo, los ha enfrentado. La consideración del rol de servicio (servant role) del bibliotecario disminuye la importancia y relevancia de su trabajo a los ojos de los docentes. Farber (1999a) afirma que los profesores reconocen que los estudiantes necesitan utilizar lo más provechosamente posible los recursos de información, pero entienden que ésa es su responsabilidad, atribuyendo a los bibliotecarios un papel de utilidad pero de subordinación. Hutchins (2005) describe a la perfección esta idea al afirmar que es indudable que los profesores consideran al bibliotecario como un profesional, pero dedicado a una función de servicio útil nunca como un igual académico. Badke (2005) considera que los profesores no respetan los roles de los bibliotecarios y que éstos ven a los profesores como arrogantemente ignorantes del funcionamiento de la biblioteca, su personal y sus herramientas.

En lo que se refiere a la necesidad de acuerdos entre ambos grupos, el papel protagonista de los docentes en la universidad supone que, frecuentemente, su trabajo sea en solitario o recurra a sus colegas y a contactos informales para obtener información (Jordan, 1998); sin embargo, el nuevo modelo pedagógico otorga a la biblioteca universitaria y a sus profesionales nuevos roles que no pueden realizar en solitario, necesitando la colaboración de otros colectivos (por ejemplo, los informáticos) pero, sobre todo, de los profesores.

También, debemos considerar que este problema ha preocupado más a los bibliotecarios que a los docentes y las iniciativas para la creación de una política de colaboración en la universidad han partido casi siempre de ellos y así lo corrobora numerosa bibliografía. Hardesty (1995) afirma que históricamente los bibliotecarios académicos han estado acudiendo a los profesores para que moti- 
vasen y sugirieran a sus estudiantes el uso de la biblioteca. La misma idea es defendida por Farber (1999b) quien considera necesario el desarrollo de una relación de trabajo entre ambos que forme a los estudiantes y docentes para una utilización más eficaz de los recursos de información disponibles. Otros autores (Chu, 1997; Iannuzzi, 1998; Winner, 1998; Hardesty, 1999; Raspa y Ward, 2000; Peacock, 2001; Jenkins, 2003), han planteado la colaboración como la clave para el éxito de la formación en los alumnos a lo largo de su recorrido académico y como ciudadanos, capacitándolos para el aprendizaje a lo largo de la vida.

Es un hecho frecuente que la literatura refleje reticencias e incluso un cierto antagonismo. Marchant (1969) hace una interesante y dura reflexión al afirmar que el nacimiento de la biblioteconomía moderna ha constituido el primer conflicto importante entre los dos grupos en la medida en que las bibliotecas universitarias de los siglos XVIII y XIX se orientaban más a los profesores, lo que ha cambiado a partir del momento en que éstas han atraído la atención de los estudiantes, y muchos docentes lo han interpretado como una amenaza a su poder sobre los procesos de aprendizaje de los alumnos, y se resienten y resisten a todo lo que parece disminuir su control o sus cometidos. El autor considera que el propio libro es una fuente potencial de competencia, puesto que puede sustituir al profesor como enseñante que se siente humillado por tener que pedir ayuda a los bibliotecarios con menor estatus que él. Además, muchos consideran que la biblioteca existe esencialmente como apoyo de sus proyectos de investigación, por lo que se muestran reacios a aceptar normas y pautas relativas a la utilización de los recursos de información disponibles en ella y que los impide utilizarlos como si fueran suyos.

Jenkins (1997) afirma que las relaciones entre ambos habitualmente se consideran "adversarias y antagónicas» debido a la existencia de diferencias entre los valores y las creencias de ambos colectivos.

No obstante, el éxito de las acciones de formación de usuarios y el impacto de los recursos electrónicos de información han contribuido al reconocimiento por parte de muchos profesores del papel del bibliotecario como formador (Hardesty, 1995; Farber, 1999b), y han ayudado a que la consideración del trabajo bibliotecario y la toma de conciencia en la necesidad de establecer acuerdos con los profesores sea ya una realidad en algunos países, como en Estados Unidos; sin embargo, no sucede lo mismo en otros, donde los bibliotecarios todavía necesitan ser aceptados, obtener la confianza del personal académico, y reafirmar la idea del establecimiento de acuerdos dinámicos en los campos de docencia y aprendizaje que implican anular las barreras existentes entre ambos.

Todas estas consideraciones nos han llevado a plantear este trabajo con el objetivo de delimitar el nuevo rol del bibliotecario universitario en la sociedad del conocimiento que ha variado los usos profesionales, tanto de los bibliotecarios como de los usuarios, y obliga a una estrecha colaboración entre ambos. Un primer paso consiste en detectar y analizar las percepciones que los docentes tienen acerca de la biblioteca de su institución, así como de sus profesionales, para poder planificarla de forma eficaz. No debemos olvidar que como resultado 
del trabajo colaborativo entre profesores, bibliotecarios y estudiantes, se formará una comunidad en la que todos se enriquezcan aprendiendo unos de otros. Entendemos que los bibliotecarios, para mejorar la percepción que de ellos tienen los profesores, deben desarrollar un papel más dinámico y activo en la misión educativa de las universidades, con un liderazgo más agresivo y conocer mejor las cuestiones relativas a la educación, para poder estar tan comprometidos con ella como con la biblioteconomía. En este sentido se expresan varios autores (Hardesty, 1995; Winner, 1998; Farber, 1999b; Breivik, 1989).

\section{Metodología}

Para detectar las percepciones que de la biblioteca universitaria y sus profesionales tienen los profesores, se ha procedido a una exhaustiva revisión cronológica de estudios empíricos que nos permita obtener una visión de conjunto y extraer unas conclusiones de cara a una nueva política universitaria que considere a la biblioteca aún más inmersa en el proceso docente de lo que ha estado hasta ahora. Los trabajos que se han escogido para efectuar nuestro análisis proceden del ámbito anglosajón por ser éste donde han proliferado más estos estudios. Además, todos ellos son considerados muy representativos y un referente para otros trabajos recibiendo numerosas citas por parte de varios autores estudiosos del tema. Hemos comenzado por la década de los setenta por constituir un punto de inflexión en estos estudios. No obstante, el análisis es más exhaustivo en la década del 2000 por considerar que la actualidad de sus conclusiones las convierten en más interesantes de cara a una planificación de nuevas estrategias de actuación por parte de los bibliotecarios.

Los estudios analizados se han llevado a cabo a través de encuestas y entrevistas personales o grupales que no siempre contemplan las mismas variables lo que dificulta realizar un análisis comparativo entre todas ellas.

\section{Percepciones de los profesores sobre los bibliotecarios a través del análisis de diversos estudios empíricos}

Como introducción a este apartado, resulta clarificadora la afirmación siguiente: "Las relaciones entre los bibliotecarios y los profesores de universidad se han descrito de forma variopinta, unas veces como eternos enemigos, otras como armoniosas y de buen hacer, y otras con visiones dispares y de comunicación inadecuada. Los docentes no están todos de acuerdo en que los bibliotecarios sean sus iguales académicamente ni que tengan su mismo rango/estatus.» (Church, 2002, p. 22).

Durante varias décadas se han realizado numerosos estudios, sobre todo en el ámbito anglosajón, con el objetivo de analizar los servicios y actividades de las bibliotecas en las instituciones de enseñanza superior para determinar la con- 
sideración de los profesores sobre el estatus de los bibliotecarios y la calidad de los servicios que prestan, así como para determinar la existencia o no de una interacción entre ambos.

\section{Década de los setenta}

En 1977, Davis y Bentley (1979) realizaron una investigación a partir de una encuesta enviada a los profesores de tres instituciones de enseñanza superior localizadas en Worcester (Massachussets, USA): Clark University, el College of the Holly Cross y el Worcester Polytechnic Institute (WPI). El objetivo del estudio era realizar, a través de una serie de variables, una exploración estadística acerca de las percepciones de los profesores hacia su biblioteca. Las variables independientes fueron: la filiación institucional, el área de enseñanza, la posición académica y la antigüedad en la institución. Las variables dependientes: las respuestas de los profesores a 23 cuestiones. La encuesta estaba estructurada en tres partes: caracterización, utilización de la biblioteca y evaluación de la misma. Las respuestas dieron como resultado la verificación de la importancia que los profesores conceden a: utilización de, percepciones sobre y actitudes para con sus bibliotecas. Debido a la relativa homogeneidad de la población (compuesta por 484 docentes) se seleccionó, en cada institución, una muestra aleatoria del $25 \%$ de profesores con dedicación plena (total 121). La tasa de respuesta fue del $66 \%$ en la Clark University, del 68\% en el WPI y del $82 \%$ en el College of the Holy Cross. Las conclusiones fueron las siguientes:

Se constataron diferencias significativas en la tasa de satisfacción por área, temas, y del tiempo en la institución: los profesores de Ciencias presentan la tasa más elevada, lo que puede ser un reflejo de la naturaleza más compacta de la literatura científica comparada con la de las Humanidades y Ciencias Sociales.

Existe una relación entre la satisfacción de los profesores y la importancia que atribuyen al apoyo del personal bibliotecario a sus temas concretos: los menos satisfechos son los que dan menos importancia a ese apoyo.

Por otro lado, los profesores menos satisfechos son aquellos cuya antigüedad en la institución es menor, lo que puede explicarse, entre otras razones, porque los más recientes están menos familiarizados con la biblioteca y sus servicios; además, pueden provenir de instituciones con bibliotecas más potentes. Las autoras sugieren que sea este último colectivo donde los bibliotecarios deben centrarse más y concentrar en ellos sus esfuerzos tanto en relaciones públicas como de servicio.

Finalmente, se recomienda que los bibliotecarios actúen de forma que atraigan la atención de los profesores hacia la biblioteca y sus recursos: realización de workshops, asistencia a reuniones de Departamento, utilización de medios de comunicación escrita, participación en las clases. Las autoras insisten en el fortalecimiento de las relaciones entre ambos.

En esta década aún no existe una gran preocupación por las relaciones profesor/bibliotecario que permanecen como colectivos claramente diferenciados y 
bastante aislados entre sí, pero constituye un punto de arranque para planteamientos futuros.

\section{Década de los ochenta}

A partir del inicio de los años ochenta, los bibliotecarios universitarios mostraron un interés creciente por las percepciones que tienen los profesores sobre su papel en la universidad y realizaron varios estudios para obtener información sobre ello. Debido a ese interés y a la escasez de datos al respecto, Cook (1981) decidió realizar un estudio en la Southern Illinois University Canbondale (SIU-C) (Illinois, USA), para el que se envió una encuesta a una muestra aleatoria del $50 \%$ de los profesores a tiempo completo. Se mandaron 507 cuestionarios y la tasa de respuesta fue del $75,7 \%$.

De todas las respuestas recibidas, el 63\% de los profesores afirma utilizar la biblioteca una o más veces por semana; el mismo porcentaje considera la colección indispensable para sus actividades de enseñanza y de investigación (para el 75\% es muy importante o indispensable).

En cuanto a los cuestionados sobre si los bibliotecarios deben tener el mismo estatus del profesorado, el 57\% respondió afirmativamente y el 43\% negativamente (el 58\% lo atribuyó a una escasa actividad docente, el $40 \%$ a una escasa actividad investigadora y de publicación, el 13\% a un servicio insuficiente, y el $27 \%$ a insuficiente nivel académico).

Las prioridades por orden de importancia en la consideración de los profesores respecto a los bibliotecarios son: el $85 \%$ considera prioritario el papel de servicio (apoyo), seguido de la investigación (el 8\%), de la enseñanza (el 5\%) y de organización y gestión de la biblioteca (el 2\%).

Las conclusiones del estudio confirman lo siguiente:

Existe gran confusión por parte de los profesores en cuanto al uso de la biblioteca por sus alumnos. Muchos indicaron que desconocían cómo son atendidos o instruidos por parte de los bibliotecarios cuando solicitan acceso a la información. En este sentido, la autora considera muy importante que los profesores asistan a workshops organizados por la biblioteca para presentarles sus recursos y servicios. También considera que los bibliotecarios deben presentar esos servicios a alumnos y los docentes, puesto que necesitan conocer las bibliografías temáticas y otros materiales que los bibliotecarios ponen a su disposición.

Los profesores no ven con malos ojos el que los bibliotecarios realicen investigación pero la restringen a temas del área de biblioteconomía, aunque muchos consideran que eso puede traducirse en una disminución en su dedicación a la prestación de servicios. Están de acuerdo en que se les conceda tiempo para que lo puedan hacer, así como que se les atribuya el mismo estatus, puesto que son parte integrante de la universidad.

Los temas tratados en este trabajo despiertan gran interés entre la comunidad bibliotecaria que ve necesario continuar en esa línea y realizar nuevas investigaciones que ayuden a planificar nuevas estrategias. 
A principios de la década, Budd y Coutant (1981) continúan los trabajos iniciados por Cook (1981) y realizan, en la Southeastern Louisiana University (SLU) (Luisiana, USA), una encuesta cuyo cuestionario se basa en el aplicado anteriormente por ese autor. La población diana la constituyó la totalidad de los profesores (292) y la tasa de respuesta fue del 51,9\%, de las cuales el 55\% declaran utilizar la biblioteca una vez o más por semana, mientras que el $45 \%$ una vez o menos por mes. Según estos autores la cuestión de la frecuencia de utilizaciones relevante pues está relacionada con otras respuestas dadas.

La importancia de la colección de la biblioteca es más elevada para los profesores de Humanidades y Educación que para los de Gestión, Ciencias y Tecnología. El 78\% de las respuestas consideran la colección muy importante o indispensable para las actividades de enseñanza y de investigación.

El apoyo de los bibliotecarios se considera muy importante o indispensable para el $65 \%$ de respuestas.

En cuanto a la contribución de los bibliotecarios a la formación de los estudiantes, los profesores de Humanidades y de Educación lo consideran más importante que los profesores de Ciencias y Tecnologías.

Sobre la cuestión de la investigación, el 35\% están de acuerdo en que los bibliotecarios puedan realizar investigación, pero únicamente en temas prácticos relacionados con la mejora de los servicios y de las colecciones.

Los bibliotecarios son considerados como profesionales por la mayoría de los profesores, el $70 \%$ opina que deberían tener el mismo estatus que el profesor. De entre aquellos que consideran que los bibliotecarios no deberían tener ese estatus, el porcentaje más significativo, el $74 \%$, lo justifica por una contribución insuficiente a la enseñanza. Otros alegan una escasa contribución a la investigación, así como insuficiente formación; sin embargo, están de acuerdo en que deberían tener los mismos salarios, mientras que algunos proponen una progresión profesional con títulos semejantes a los de los profesores (assistant librarian, associate librarian, etc.).

Los autores establecieron una relación de concordancia entre frecuencia de utilización de la biblioteca/atribución del estatus del profesor a los bibliotecarios, y entre frecuencia/importancia atribuida a la colección. Concluyen que cuestionarios como los realizados en la SLU y, anteriormente en la SIU-C, son instrumentos muy útiles que los bibliotecarios deben utilizar para saber las percepciones de los profesores y, además, los resultados insinúan áreas en las que el servicio puede mejorarse y otras donde las relaciones con los profesores pueden estrecharse. Recordemos que Cook (1981) había ya recomendado la necesidad de realizar estudios comparativos para obtener un amplio conocimiento sobre las actitudes del profesorado hacia los bibliotecarios.

El tema continúa con un interés creciente y en 1985, Divay y otros (1987) parten de los estudios y sugerencias anteriormente citados para realizar una investigación en la University of Manitoba, una de las mayores universidades de Canadá. La encuesta se diseñó con el objetivo de descubrir las percepciones de 
los profesores hacia los bibliotecarios y determinar el alcance y la naturaleza de la interacción entre ambos. Para ello se envió por mail a la totalidad de los profesores con dedicación plena (1095). La tasa de respuesta fue del 59\%.

Los aspectos analizados fueron: tipo de contacto profesor-bibliotecario (dentro y fuera del entorno de la biblioteca); percepción de la utilidad de los bibliotecarios; percepción de la importancia de los antecedentes de los bibliotecarios en materia académica y percepción del estatus de los bibliotecarios en la universidad. En líneas generales, los resultados arrojan estas cifras:

Respecto a la frecuencia de contactos con el bibliotecario, éstos se limitan prácticamente al envío de alumnos a la biblioteca para realizar los trabajos que determinan los profesores: el 30\% afirma dirigir a la biblioteca a sus estudiantes diariamente o varias veces al mes; el $42 \%$ una vez al mes o varias al año y el $20 \%$ nunca. El $90 \%$ concede mucha importancia al servicio de información y referencia (el 62\% considera importante o muy importante el papel del bibliotecario en prestación de información sobre la biblioteca; el 38\% sobre nuevas publicaciones).

En lo que se refiere a la consideración del apoyo que la biblioteca puede prestar a la formación académica de los alumnos, el 63\% de profesores consideran importante o muy importante (el 42\% considera que el bibliotecario se involucra en ello y el $21 \%$ que la repercusión es considerable).

Sin embargo, no se concede mucha importancia al papel desarrollado en el proceso educativo, únicamente el $50 \%$ consideran importante o muy importante el apoyo a actividades de docencia. La actividad de los bibliotecarios en la investigación, en la enseñanza y en la gestión, representa papeles poco importantes.

Las conclusiones del estudio apuntan lo siguiente:

Percepción mayoritaria y reiterada del papel de servicio (apoyo) de los bibliotecarios debido a su consideración como profesionales y no como académicos.

Tasa relativamente alta de la interacción entre profesores y bibliotecarios.

Perspectiva positiva sobre la utilidad de los bibliotecarios y el valor de su experiencia presente y futura en la materia.

Las recomendaciones de todos estos autores se centran en que los bibliotecarios deben realizar un esfuerzo para informar a los docentes acerca de su potencial y para ello es necesario promocionar su imagen. Del mismo modo, los profesores deben tener la plena seguridad de que la conducta de los bibliotecarios está dirigida a responder a sus necesidades de información, al mismo tiempo que tienen la responsabilidad de ayudar a concretar los objetivos a largo plazo de la universidad. En este sentido, recomiendan que la comunicación y comprensión entre ambos sea cada vez mayor, puesto que unir sus esfuerzos proporcionará educación de alta calidad, tanto a nivel de grado como postgrado. El aumento de la interacción a través de consultas, comités y talleres podría dar lugar a una percepción diferente sobre el rol del bibliotecario como investigador, educador y responsable de gestión, lo que mejoraría sus posibilidades para ser plenamente aceptados por sus compañeros profesores. 
La cuestión de la importancia que para los bibliotecarios tiene cómo son considerados por los profesores y la necesidad de una estrecha colaboración entre ambos se ratifica, cada vez más, en la literatura de este período. Así en 1989, Oberg y otros (1989) parten de la ambigüedad y de la invisibilidad del papel del bibliotecario para determinar las percepciones que de ellos tienen los profesores. Para estos autores: "...en cómo los bibliotecarios son vistos por este grupo de usuarios influye no sólo su estatus, sino también su grado relativo de aislamiento de los centros de poder del campus, cómo se financia la biblioteca, y con qué intensidad y éxito se explotan sus recursos.» (p. 216). Refieren los estudios anteriores, reafirmando la necesidad de continuar realizando análisis con las mismas características porque los bibliotecarios tienen la responsabilidad de «...reexaminar constantemente los principios de su campo.» (p. 216). El estudio se llevó a cabo en el Albion College (Michigan, USA). El cuestionario se envió a la totalidad de los profesores con dedicación plena (109), con una tasa de respuesta del 80\%).

Del mismo modo que el estudio de Divay y otros (1987) situaba la mayor frecuencia de contactos entre los profesores y los bibliotecarios en el apoyo de referencia (el 90\%), lo mismo sucede en este estudio (el 86\%). Interesante es verificar que los profesores que realizan su investigación orientada a la publicación revelan un nivel de contacto con los bibliotecarios más elevado (el 88\%) que los que la hacen orientada a la enseñanza (el 65\%).

Consideran importante o muy importante el papel de la biblioteca en prestación de información el 93\% de respuestas, el 76\% sobre nuevas publicaciones y el $74 \%$ en el apoyo a actividades de docencia.

Aquellos que no están de acuerdo con la atribución del estatus del profesor a los bibliotecarios (el 71\%), apuntan los mismos motivos que en el estudio de Budd y Coutant (1981), a saber, insuficiente actividad de enseñanza y de investigación, seguida por una formación inadecuada. Los profesores ven a los bibliotecarios como profesionales que garantizan un amplio conjunto de servicios muy valorados pero no los consideran centrales para las misiones de enseñanza y de investigación de la institución.

Entre las conclusiones de este estudio, una de las más destacables es que las percepciones que los profesores tienen de los bibliotecarios están influidas por la naturaleza y por la frecuencia de los contactos que mantienen con ellos. Los profesores que utilizan la biblioteca con más frecuencia son los que mantienen más contactos con los bibliotecarios. En este sentido, y en la misma línea del estudio de Budd y Coutant (1981): “...cuanto mayor es su contacto con los bibliotecarios, más probable es que los acepte como iguales» (p. 224).

También se afirma que una de las dificultades más importantes a que los bibliotecarios se enfrentan para clarificar su imagen es la ausencia de consenso en la profesión acerca de lo que este colectivo debe hacer. Se trata de un problema de identidad profesional. También los estereotipos que caracterizan a los bibliotecarios como gatekeepers y a las bibliotecas como almacenes de libros, constituyen barreras que deben superarse. Los resultados muestran que los usuarios centran su atención en las actividades más visibles. 
Este estudio refuerza la idea de que los cambios por los que están pasando las bibliotecas universitarias han obligado a sus profesionales a desempeñar nuevas funciones que, en palabras de los propios autores: «...Lo que hacen en realidad (...) es intelectual, abstracto y central para el proceso de comunicación erudita" (p. 225), sin que se les haya comunicado de forma clara a los usuarios y, entre ellos, a los profesores. Por tanto, es necesario que los bibliotecarios comuniquen una imagen más clara de quiénes son y qué es lo que hacen. De lo contrario perpetúan su aislamiento en la toma de decisiones en consejos institucionales, contribuyen a la infrautilización de sus habilidades y conocimientos, empobrecen los contactos entre usuarios-bibliotecarios y usuario-colección y obstaculizan sus propios esfuerzos para involucrarse más en la educación.

\section{Década de los noventa}

Crece la idea de que una estrecha colaboración profesor/bibliotecario incide directamente en una visión positiva del profesor para con su biblioteca. Los bibliotecarios son conscientes de que han de ser más visibles en la institución y continúan realizando trabajos que delimiten su perfil profesional.

En 1990, Ivey (1994) realizó una encuesta en la estadounidense Memphis State University (MSU). En este estudio, el autor considera que «Los bibliotecarios académicos muestran una creciente preocupación sobre la forma en que son percibidos por sus colegas profesores» (p. 69). Tiene en consideración los estudios anteriormente citados sintetizando las contribuciones de cada uno. De hecho, se incluyen cuestiones ya realizadas por ellos.

El cuestionario se envió a la totalidad de los 880 profesores con dedicación plena. La tasa de respuesta fue del $45 \%$. Esta encuesta difiere de las anteriores en que para determinadas cuestiones se distinguen los profesores que hacen investigación esencialmente orientada a la enseñanza de los que la hacen orientada a la publicación. De este modo, mientras que para el $79 \%$ de los primeros los bibliotecarios son importantes o muy importantes en la prestación de información sobre nuevas publicaciones en su campo, sólo para el 30\% de los segundos los bibliotecarios son importantes o muy importantes. En cuanto a las actividades docentes, el 53\% de los profesores que hacen investigación orientada a la enseñanza los consideran importantes o muy importantes, mientras el porcentaje es del $50 \%$ para los que la realizan orientada a la publicación.

Respecto a las preguntas que no distinguen a los profesores según su orientación a la docencia o a la publicación, los resultados son los siguientes: el 44\% de respuestas afirman dirigir al estudiante al bibliotecario diariamente o varias veces al mes. El 33\% lo hacen una vez al mes o varias veces al año y el $17 \%$ nunca lo hacen.

Sobre la contribución de los bibliotecarios a la formación académica de los estudiantes, el $62 \%$ de los profesores consideran que tienen una considerable 
repercusión. De estos, el 38\% considera que los bibliotecarios están involucrados y el $23 \%$ que tienen una repercusión considerable.

Sobre el orden de prioridad de las diversas funciones, el 57\% considera prioritario el papel de servicio (apoyo), seguido de la investigación (el 40\%), de la enseñanza (el 16\%), de la gestión (el 15\%) y de la administración (el 8\%).

La mayor frecuencia de contactos bibliotecarios/profesores se produce en el servicio de referencia (el 89\%), seguido de la búsqueda de recursos de información electrónicos (el 60\%), la gestión y desarrollo de las colecciones (el 41\%), la formación de usuarios (el 24\%) y por los asuntos de política bibliotecaria (el 10\%).

Este autor considera que si los profesores tienen pocos contactos con los bibliotecarios «...cabe preguntarse cómo se puede entender y apreciar las contribuciones de los bibliotecarios a la comunidad académica.» (p. 81). También insiste en la necesidad de que los bibliotecarios desarrollen acciones de marketing sobre sus competencias considerando que «Sólo cuando hagan visible lo invisible serán considerados como iguales por sus colegas.» (p. 81).

El trabajo que se analiza a continuación se llevó a cabo por Dilmore (1996), el cual hace una aportación interesante al constatar que los autores que anteriormente habían analizado el papel de la comunicación en las bibliotecas se habían limitado a estudiarla desde dentro y partiendo de la consideración de la posible relación entre la comunicación y las percepciones de los profesores sobre los servicios de la biblioteca y su utilización, o cuando estudiaron la comunicación externa se limitaron a la publicitación de servicios y otros asuntos de relaciones públicas. El autor desarrolló un estudio en nueve colleges de New England (USA) con dos objetivos:

«... determinar si la interacción se llevaba a cabo entre los bibliotecarios académicos y el profesorado y cómo se estaba desarrollando esa interacción.» (p. 275). «... explorar las posibles relaciones entre bibliotecario/profesorado y las percepciones de estos últimos - y uso de- los servicios bibliotecarios.» (p. 275).

En este sentido, examinó el número de actividades de contacto (outreach) mantenidas durante dos semanas de trabajo y para ello solicitó a los bibliotecarios mantener un registro diario de los contactos con los profesores de cada centro. Además, envió a ambos colectivos un cuestionario, distinto según si se trataba de bibliotecario o profesor aunque con cuestiones comunes, sobre actividades de contacto. Comparó los resultados para determinar en qué medida las percepciones de los bibliotecarios coincidían con las de los profesores. En lo concerniente a los profesores (total: 1.397), se enviaron 511 cuestionarios y la tasa de respuesta fue del $47 \%$, con una variación entre el 34\% y el 63\%, dependiendo del centro. Respecto a los bibliotecarios, se enviaron 59 cuestionarios y la tasa de respuesta fue del $85 \%$ (se recibieron 50).

El número de contactos profesor/bibliotecario constatados en el registro diario arrojan el resultado de que 599 profesores (el 43\%) mantuvieron contactos con los bibliotecarios. 
Los resultados de la encuesta a bibliotecarios apuntan a que éstos atribuyen puntuaciones más bajas a las actividades de contacto desarrolladas que los profesores, con excepción de aquellas «... que están más directamente asociadas a la prestación efectiva de servicios de la biblioteca. Estas áreas fueron las siguientes: en calidad de expertos, proporcionando asesoría a los estudiantes y profesores acerca de cómo utilizar los recursos de información y proporcionando instrucción formal en el uso de la biblioteca.» (p. 279).

Los datos permiten establecer una correlación positiva entre la frecuencia de los contactos y la percepción de los profesores sobre los servicios de la biblioteca, lo que complementan los obtenidos por Ivey (1994) y los del estudio de Oberg y otros (1989).

Los resultados de la encuesta a los profesores fueron los siguientes:

En cuanto a la percepción sobre la calidad de los servicios de la biblioteca, no existen diferencias por área científica (estudios profesionales, artes liberales, ciencias y ciencias sociales); sin embargo, estas diferencias sí son significativas en la utilización de los servicios.

Existencia de una correlación positiva entre el número de años en la institución y la percepción sobre los servicios de la biblioteca, lo que confirma el estudio de Davis y Bentley (1979).

Existencia de una correlación positiva entre la calidad de la colección y las percepciones y utilización de los servicios de la biblioteca. Uno de los resultados más interesantes del estudio fue que una de las bibliotecas más activas en acciones de contacto con los profesores recibió la puntuación más alta. Este resultado corrobora que la existencia de una política de comunicación es determinante para una percepción favorable de los profesores respecto a los servicios de la biblioteca y de los bibliotecarios.

En 1996, dado que el asunto continuaba de actualidad, Byron (1997) realizó otro trabajo en el College of Arts and Sciences de la University of North Texas (UNT) de Estados Unidos «...para explorar las percepciones del profesorado acerca de la valía de los bibliotecarios para la enseñanza y la investigación y para aprender más sobre qué permitiría al profesorado hacer un mayor uso de las bibliotecas de la UNT.» (p. 3).

En esta ocasión la metodología de trabajo utilizada consistió en la creación de dos grupos de discusión (focus groups) con 8 profesores cada uno seleccionados de cuatro áreas diferentes: Humanidades, Ciencias Naturales y Físicas, Programas Profesionales y Ciencias Sociales.

La elección se debió al hecho de "...esta metodología ofrece una imagen más rica y comprensible que los métodos más tradicionales de encuestas.» (p. 3). Los temas debatidos en las reuniones fueron:

- Acceso a los recursos electrónicos.

- Acceso a las revistas científicas.

- Papel de la biblioteca en el apoyo a los estudiantes. 
Las conclusiones de los debates realizados subrayan una percepción favorable de los profesores sobre el apoyo de las bibliotecas a la enseñanza y a la investigación, así como la preocupación en el acceso a los recursos electrónicos y a las publicaciones periódicas.

Se formularon dos recomendaciones:

- «Fortalecer el programa actual de enlaces bibliotecarios para mantener directamente informado al profesorado de cómo acceder a los recursos electrónicos de sus respectivas disciplinas, el estado de la colección de revistas, y las opciones disponibles para acceder a las publicaciones sin necesidad de llevárselas físicamente.» (p. 3).

- «Continuar los esfuerzos para integrar todos los recursos electrónicos dentro de las infraestructuras informáticas del campus.» (p. 3).

\section{Década de los 2000}

Esta década viene marcada por el uso generalizado del documento electrónico y de la denominada "tecnología internet» que marcará la labor del bibliotecario y de los usuarios, así como la percepción de unos sobre otros.

Entre febrero y marzo del 2000 tuvo lugar otra investigación, a cargo de Feldman y Sciammarella (2000), en la City University of New York (USA), que utilizó la metodología de la encuesta por cuestionario.

Se desarrollaron dos cuestionarios, el primero destinado a los bibliotecarios para confirmar sus sentimientos respecto a su consideración profesional, y el segundo a los profesores para comprender mejor sus percepciones sobre los bibliotecarios y la biblioteconomía.

Se enviaron 425 cuestionarios a los profesores y 75 a los bibliotecarios. La tasa de respuesta de los primeros fue del 37\% y de los segundos del 69\%.

Los resultados relativos a los profesores fueron los siguientes:

- el $64 \%$ de los profesores que han respondido declara no utilizar el servicio de formación de usuarios de la biblioteca (el 21\% porque desconocía su existencia, el $12 \%$ porque considera que los estudiantes deben ya saber cómo buscar y escribir un texto académico, el 25\% porque los estudiantes deben buscar el apoyo del profesor, y el $25 \%$ porque indican a los estudiantes el contacto directo con el bibliotecario);

- el 63\% declara pedir a los estudiantes la realización de tareas y trabajos que implican el uso de la biblioteca y que lo hace con el objetivo de que los estudiantes acudan a ella. Los autores de la encuesta consideran este hecho importante «...porque un requerimiento para localizar libros o un artículo en materias concretas puede ser un comienzo para que los estudiantes usen la biblioteca, en particular para cursos donde no se proporcionan.» (p. 494); 
- del 36\% de profesores que declaran llevar a los estudiantes a la biblioteca para clases de formación bibliográfica, el 75\% responde que la interacción verbal con los bibliotecarios es necesaria para que la clase sea productiva;

- únicamente el 35\% de los profesores afirman haber asistido a sesiones de formación en recursos de información electrónicos impartidas por la biblioteca;

- a pesar de no participar en sesiones de formación, el 69\% afirma mantener contacto con los bibliotecarios en el ámbito de sus actividades de investigación o de la de sus estudiantes y el $82 \%$ considera que el bibliotecario los puede ayudar a encontrar información necesaria para sus proyectos de investigación.

Los resultados de la encuesta a los bibliotecarios fueron:

- el $80 \%$ de las respuestas consideran que su estatus de no docente no es adecuado, debido al hecho de que aseguran la docencia de las metodologías de investigación;

- el 84\% considera que su reconocimiento como profesores sería importante para promover su estatus en la comunidad académica;

- el $67 \%$ posee un grado de máster;

- el $44 \%$ ha participado en proyectos de investigación sobre su área o ha publicado en los últimos cinco años;

- el $77 \%$ ha asistido a acciones de formación, seminarios, etc., en los dos últimos años;

- el $92 \%$ considera que los profesores no son conscientes de la naturaleza del campo de las ciencias documentales;

- el 94\% acredita que los profesores que asignan trabajos de investigación no están familiarizados con el uso de herramientas actuales disponibles para sus estudiantes en la biblioteca. Esta idea contrasta con los resultados de la encuesta a los profesores ya que el $90 \%$ declara conocer los recursos de información disponibles en la biblioteca para sus alumnos.

Es importante señalar que ambos autores afirman el interés común que ambos colectivos tienen en los estudiantes, así como el hecho de que muchos profesores son conscientes de la importancia que tiene para sus alumnos la adquisición de competencias informacionales y que los bibliotecarios pueden ayudarlos a utilizar la biblioteca; sin embargo, tienen algunas dudas sobre la posibilidad de que desarrollen actividades de investigación o de enseñanza con ese objetivo.

Asimismo, destacan la ausencia de comunicación entre ambos, aspecto que tiene consecuencias en la formación de usuarios, en el trabajo de referencia y que para ser superado necesita que los bibliotecarios «...eviten la tentación de la retirada (...) en soledad como si se tratara de una comunidad de mártires asediada (...). Y puesto que los asuntos de los bibliotecarios están en un buen momen- 
to, son ellos los que tendrán que tomar la iniciativa en la apertura de líneas de comunicación y los que deben, según su costumbre, sugerir y obtener comentarios de los profesores.» (p. 399).

Poco a poco las percepciones van cambiando y aunque predomina por parte del profesor su visión del bibliotecario como "servant role», se comienza a considerar su papel en asuntos relativos a su implicación en docencia y en investigación. Así, en marzo del mismo año, se realizó otra investigación, esta vez por parte de Ducas y Michaud-Oystryk (2003), en la University of Manitoba (Canadá) con el objetivo de analizar la interacción entre ambos grupos y los papeles futuros para los bibliotecarios. Se concretaron cinco áreas de actuación: enseñanza/formación, servicios de información, tecnologías de información, investigación y colecciones. Este trabajo se llevó a cabo pasados 15 años del realizado en 1985 por Divay y otros (1987) por lo que conviene recordar que en aquel momento los profesores consideraban a los bibliotecarios como profesionales con una misión de servicio (apoyo), y los roles de investigación, enseñanza y gestión eran poco reconocidos. Para evidenciar la existencia de eventuales cambios en aquella realidad, se desarrolló una nueva investigación centrada en los aspectos siguientes:

- el papel actual que los bibliotecarios están jugando en colaboración con el profesorado;

- el impacto de las contribuciones de los bibliotecarios en el trabajo universitario;

- los futuros roles de los bibliotecarios que pueden mejorar las relaciones bibliotecario/profesorado.

Los autores señalan que en estos quince años se han producido algunos cambios que afectan a los cometidos y responsabilidades de los bibliotecarios de educación superior: explosión de los recursos de información disponibles en diferentes formatos, transición de un entorno de información esencialmente en papel hacia otro esencialmente electrónico y abundancia de innovaciones tecnológicas. Para ellos, los bibliotecarios «...están desarrollando —y continuarán haciéndolo- un papel crítico en la evaluación, análisis y filtrado de la información.» (pp. 55-56).

La investigación se desarrolló a través de una encuesta por cuestionario enviada a los 1.400 profesores con dedicación plena con una tasa de respuesta del 52\%, con la siguiente distribución por áreas científicas: Humanidades y Ciencias Sociales (el 36\%), Ciencias de la Salud (el 42\%) y Ciencias Puras y Aplicadas (el 20\%).

La primera parte del cuestionario se destinaba a determinar el nivel de interacción y de colaboración profesores-bibliotecarios en cada una de las áreas de actuación anteriormente mencionadas.

En cuanto a enseñanza-formación, el 79\% de las respuestas declararon no mantener ningún tipo de contacto. Casi la mitad consideran inadecuado pedir a 
un bibliotecario que garantice actividades de enseñanza en los cursos o que asegure actividades de formación de usuarios; el 28\% respondieron desconocer que los bibliotecarios desarrollaban esas actividades, reforzando la necesidad de que éstos promovieran (marketing) sus competencias.

Respecto a este tema, se verificó una gran discrepancia por áreas científicas: el 58\% de los profesores de Ciencias Puras y Aplicadas no considera adecuado que los bibliotecarios enseñen o aseguren formación de usuarios; para Humanidades y Ciencias Sociales el porcentaje es del 43\% y para Ciencias de la Salud del 38\%.

Sobre los servicios de información, el 88\% de los profesores declaró haber solicitado ayuda a un bibliotecario para encontrar la información que necesitaba. También aquí existen variaciones según el área: el 83\% para Ciencias Puras y Aplicadas y el 91\% para Humanidades y Ciencias Sociales. Del 12\% que declaró nunca haber solicitado apoyo, el 58\% afirmó no necesitarlo, el $21 \%$ raramente visitar la biblioteca y el $20 \%$ nunca haber pensado en esa posibilidad.

Respecto a las tecnologías de información, sólo el 33\% respondió haber solicitado apoyo: de ellos, el 18\% de los profesores pertenecía a Ciencias Puras y Aplicadas, el 35\% a Ciencias de la Salud y el 39\% a Humanidades y Ciencias Sociales.

Del $67 \%$ que declaró no haber solicitado nunca apoyo en asuntos relacionados con las tecnologías de información, la justificación más frecuente fue que desconocía que el bibliotecario los podía ayudar. Únicamente el 12\% pensaba que los bibliotecarios tenían insuficiente capacidad o experiencia.

Acerca de recurrir a los bibliotecarios en asuntos relativos a la investigación, sólo el $7 \%$ respondió haberlo hecho, y aquí no se verifican diferencias entre áreas científicas. De éstos, el $65 \%$ lo ha hecho a través de la búsqueda de literatura, el 26\% a través de la búsqueda de información y el 22\% como socio (partner) en un proyecto de investigación.

Del 93\% que declaró no haber colaborado con un bibliotecario en un proyecto de investigación, más de la mitad contestó que nunca había pensado en esa posibilidad, el 23\%, respondió que la investigación en colaboración no es parte de la cultura institucional y el $20 \%$ declaró no disponer de tiempo. Sólo el $17 \%$ pensaba que los bibliotecarios tenían insuficiente capacidad y experiencia o que no eran apropiados para ser parte de un proyecto de investigación.

Los resultados por área científica revelan diferencias: el porcentaje de aquellos que declararon que nunca habían pensado en la posibilidad de colaborar es del 35\% en Ciencias Puras y Aplicadas, del 50\% en Humanidades y Ciencias Sociales y del $62 \%$ en Ciencias de la Salud. Los primeros justifican ese hecho por considerar que los bibliotecarios no tienen las competencias o los conocimientos necesarios y también por considerar inadecuado que se integren en un proyecto de investigación.

En cuanto al último punto, el desarrollo de las colecciones, el 38\% afirmó haber mantenido contacto con la biblioteca: de estos, el 23\% de Ciencias de la Salud, el 45\% de Ciencias Puras y Aplicadas y el 54\% de Humanidades y Ciencias Sociales. 
En este estudio se procuró evaluar el impacto de las contribuciones de los bibliotecarios en las cinco dimensiones. Los datos obtenidos a partir de las respuestas al cuestionario revelan un impacto muy positivo de los bibliotecarios en la investigación (el 96\%), en los servicios de información (el 94\%), en las tecnologías de información (el 91\%), en las colecciones (el 89\%) y en la enseñanza/ formación (el 77\%).

Por último, a través de un conjunto de cuestiones de ámbito general, los autores evaluaron el papel del bibliotecario en la comunidad académica y los profesores estimaron el rol del bibliotecario en el siguiente orden de importancia decreciente: servicios de información (el 84\%), colecciones (el 80\%), tecnologías de información (el 69\%), investigación (el 60\%), y enseñanza/instrucción (el 50\%).

A pesar de haber transcurrido 15 años entre un análisis y otro, los autores consideran que «...este estudio reciente demuestra que en los años intermedios no parece haber habido un cambio importante en las actitudes y expectativas de los profesores.» (p. 72).

Dado que el estudio anterior no muestra un cambio de actitud por parte del colectivo docente muy positivo, Manuel y otros (2003) realizaron una investigación en Estados Unidos, en concreto en la New México State University (NMSU), con el objetivo de determinar los aspectos que más valoran los profesores que utilizan frecuentemente los servicios de formación bibliográfica (alfabetización informacional) con objeto de detectar aquellos que inclinen la balanza en una actitud más positiva. Seleccionaron 30 profesores para entrevistas en profundidad con el objetivo de determinar porqué utilizan en sus cursos las indicaciones del bibliotecario y qué obtienen con ello.

Además de las entrevistas, los profesores respondieron al Learning Organizations Practice Profile (LOPP), una encuesta utilizada para medir las percepciones de la gente acerca del clima organizacional, el flujo de información, desarrollo de prácticas individuales y en grupo, procesos de trabajo y los objetivos de rendimiento. La misma encuesta se envió a 100 profesores seleccionados de modo aleatorio que no utilizan la formación bibliográfica (grupo de control). La tasa de respuesta fue del 50\% para las entrevistas y del 30\% para la encuesta.

Los profesores presentan una concordancia mayor con las siguientes afirmaciones: "Tenemos una visión de nosotros mismos como una organización en la que se espera el aprendizaje y el cambio intencional; no tenemos miedo de compartir nuestras opiniones y decir lo que pensamos; eliminamos la mentalidad "nosotros/ellos"; cooperamos y colaboramos siempre que sea posible; nos tratamos unos a otros como adultos - como personas que pueden pensar por sí mismas y ser responsables; personas interesadas y que se preocupan unas por Otras (...). (p. 5)».

A través de las entrevistas y de la encuesta, los autores destacan algunos puntos sobre los que los bibliotecarios deben reflexionar para trabajar de forma más eficaz con profesores y estudiantes en la promoción de las competencias informacionales. Los agrupan en tres áreas: sentido (meaning) de la colaboración, valor de la formación bibliográfica y cultura de los profesores. 
Sobre la primera cuestión entienden que lo que no existe, y que puede ser imposible de alcanzar, son criterios que los bibliotecarios puedan utilizar con algún grado de exactitud para identificar posibles socios colaborativos. Por otro lado, se constata que los profesores expresan un fuerte sentido de valores y objetivos organizacionales y ven a sus colegas como personas con las que comparten y colaboran; sin embargo, no siempre es así en lo concerniente a su relación con los bibliotecarios.

La importancia atribuida a la formación bibliográfica no es igual para bibliotecarios que para profesores. Muchos docentes la consideran como algo a corto plazo (desarrollo de competencias que permitan realizar una tarea específica) y no a largo plazo (desarrollo de competencias de alfabetización informacional, competencias de pensamiento crítico, capacidades para el aprendizaje a lo largo de la vida) que es la consideración que tienen los bibliotecarios.

Por último, mientras la formación de los profesores queda garantizada por su especialización en un área concreta, puesto que deben mostrar un alto nivel de compromiso con la investigación en sus disciplinas para obtener las credenciales básicas necesarias para convertirse en miembros de una universidad, esto puede actuar en contra de la colaboración en la formación bibliográfica ya que:

1. los profesores piensan que los bibliotecarios no disponen de una especialización, por lo que los consideran miembros menos capaces en la educación superior;

2. los profesores no conceden gran importancia e interés a la enseñanza de competencias, como las de alfabetización informacional, por entender que corresponden más a formación global y no al currículo de sus disciplinas;

3. percepción de que las instituciones habitualmente no reconocen ni recompensan la colaboración o las contribuciones para la educación/formación global.

A partir de este momento, los trabajos relativos a las relaciones profesor-bibliotecario deja de ser objeto únicamente de las ciencias documentales (donde siguen prestándose más atención a asuntos como las expectativas de los bibliotecarios sobre los profesores, el impacto de su cultura en la formación de usuarios —alfabetización informacional- y su estatus), para pasar también a serlo de las ciencias sociales; sin embargo, no como asunto prioritario, puesto que sus investigaciones se van a centrar en cuatro temas (con mayor incidencia de los dos primeros): género y sexo en la profesión; uso de los recursos de información con el objetivo de enseñar métodos de investigación en sociología y formación de usuarios; estatus, prestigio y profesionalidad de los bibliotecarios; decisiones (políticas) sobre colecciones.

En este sentido, Christiansen y otros (2004) afirman que mientras los bibliotecarios han escrito mucho sobre sus relaciones con los profesores, este tema 
está casi ausente de la literatura de las ciencias sociales. Por este motivo, decidieron analizar «...cómo los estudios de biblioteconomía y ciencias sociales tratan el tema de las relaciones profesores-bibliotecarios, destacando los principales temas y cuestiones metodológicas.» (p. 116).

Los autores apuntan al estudio de Kotter (1999) como el más importante y completo en este tema destacando su afirmación de que los métodos utilizados en los estudios realizados hasta ese momento no permitían alcanzar conclusiones generalizables a todos las bibliotecas/bibliotecarios y recomendando la realización de análisis más sistemáticos con el objetivo de contribuir a la mejora de las relaciones entre los dos grupos.

Afirman que las encuestas realizadas hasta ahora a los docentes permiten concluir una serie de afirmaciones tales como: un nivel elevado de satisfacción con los servicios de la biblioteca; no existe un nivel elevado de colaboración; declaran estar disponibles para colaborar con los bibliotecarios pero esa voluntad no se concretiza en acción; para los profesores los bibliotecarios tienen un papel, sobre todo, de servicio y no educativo/formativo. Sin embargo, los bibliotecarios insisten en la separación entre los dos grupos y aunque reconocen la existencia de algunas experiencias positivas (desarrollo colaborativo de sitios en la Web, desarrollo curricular, integración de la alfabetización informacional en el currículo), se quejan de muchas limitaciones por parte de los profesores para una auténtica colaboración.

Los tres autores realizaron un estudio en una Universidad y un college públicos, la Texas Tech University (Texas, USA) y el Augsburg College (Minneapolis, USA), que incluyó encuestas a ocho profesores y a un bibliotecario, entrevistas a tres profesores y a dos bibliotecarios, y un focus group con ocho profesores.

Este estudio permitió concluir que existe una desconexión asimétrica (asymmetrical disconnection) entre los dos grupos, que ambos son necesarios para el funcionamiento exitoso de cualquier centro académico pero que los dos están separados. Este hecho es sorprendente para los autores debido a «...su potencial para la interacción, la colaboración, y los intereses compartidos por la calidad de la enseñanza y la investigación.» (p. 117). Esa interacción debería significar que los cambios en las prácticas de un grupo implicarían cambios en las prácticas del otro. Cada uno de ellos entiende esta desconexión de modo distinto.

Entre ambos colectivos, los que más conocen el trabajo del otro y más se esfuerzan en establecer contactos son los bibliotecarios mientras que para los profesores el papel de la biblioteca es organizar y facilitar el acceso a los recursos y a las colecciones. Esta desconexión presenta algunos problemas para los bibliotecarios que, en ocasiones, les dificulta lograr sus objetivos de trabajo siendo el más importante ayudar a los estudiantes. Sin embargo, no es así para los profesores "...no perciben ningún problema serio en las relaciones entre los dos grupos, ni tampoco se identifican las posibles consecuencias negativas derivadas de una desconexión.» (p. 118).

Para explicar este fenómeno, los autores presentan dos dimensiones: la organizacional y el estatus. En la primera, incluyen las divisiones en tiempo y físicas 
en la medida que en la mayoría de los centros los profesores están dispersos por todo el campus con horarios de trabajo más flexible y que pueden realizarlo en su despacho o en su vivienda, mientras que los bibliotecarios deben permanecer en la biblioteca y su horario de trabajo es más rígido. Además, las tecnologías de información han agravado la separación en la medida que permiten a los profesores acceder a los recursos informativos sin necesitar de desplazarse. En consecuencia, la separación física y temporal de ambos tiene impacto en la oportunidad «...para una interacción significativa y reconocimiento mutuo de experiencia y respeto colegial.» (p. 118).

Constatan también la existencia de subculturas organizacionales distintas. Las bibliotecas promueven una cultura de compartición, cooperación y colaboración y, en ese sentido, "Parte de lo que define la biblioteconomía es "llegar» a los usuarios de la biblioteca (estudiantes, profesores, y otros) para mejorar su servicio.» (p. 118). Sin embargo, la cultura de los profesores es más de aislamiento y propietaria y la colaboración se realiza en el ámbito de proyectos de investigación.

Para los profesores, los bibliotecarios no tienen un papel principal que desarrollar en las actividades de docencia o investigación y entienden que las clases son un dominio suyo. Por otro lado, el poder de cada grupo en la organización es distinto en la medida que los profesores controlan la mayoría de los órganos decisorios de la universidad lo que puede explicarse por «...el desarrollo histórico de las jerarquías académicas, las diferencias de estatus entre los bibliotecarios y profesores, y la proporción de profesores respecto a bibliotecarios en cualquier campus.» (p. 119). Una de las consecuencias es que los docentes se consideran un grupo distinto de los demás.

En cuanto a los bibliotecarios, su trabajo se destina a apoyar a los profesores y a los estudiantes, por lo que su éxito se mide por la calidad del servicio para la organización y para sus usuarios. Este hecho ayuda a explicar la razón por la que los contactos con los profesores son tan importantes. Por el contrario, el éxito del trabajo de los profesores se mide, en primer lugar, por la calidad y cantidad de investigación (las interacciones son con otros profesores) y de docencia (las interacciones son con otros profesores y con los estudiantes), lo que explica que no concedan tanta importancia a los contactos.

En cuanto al estatus, los profesores afirman que su trabajo está enfocado a la producción y diseminación de conocimiento y que los bibliotecarios realizan tareas orientadas al servicio (service-oriented). En la medida en que «...la sociedad contemporánea, en general, considera el trabajo orientado a servicios como de menor importancia que los de producción, debido principalmente a las relaciones implícitas de orden superior-subordinado que parecen inherentes a los servicios.» (p. 119), las consecuencias «... son profundas e importantes, y se refieren a las diferencias en las expectativas de tiempo de trabajo, remuneración y beneficios, poder en las políticas y problemas de todo el centro, y en términos de relaciones sociales en el campus entre los profesores y todos los demás.» (p. 120).

La feminización de la profesión también contribuye para que sea menos valorada, pues como lo han demostrado los sociólogos, las actividades que son 
mayoritariamente desarrolladas por mujeres son consideradas como menos importantes por lo que «en la medida en que las bibliotecas siguen siendo consideradas "trabajo de mujeres" y que este trabajo se considera que tiene menor valor que el tradicional "trabajo de hombres", se refuerzan las diferencias de condición de género entre los bibliotecarios y los profesores.» (p. 120).

Los autores de este trabajo insisten una y otra vez en que para solucionar el problema los bibliotecarios han de asumir nuevas estrategias entre ellas la de dar mayor visibilidad a sus funciones utilizando técnicas de promoción.

Las cuestiones de formación de usuarios son cada vez más objeto de atención por parte tanto de bibliotecarios como de profesores, en ello tiene mucho que ver la implantación del Espacio Europeo de Enseñanza Superior (EEES) que modifica la metodología de enseñanza, uno de cuyos pilares va a ser el autoaprendizaje, lo que hace imprescindible un uso racional y eficaz de los recursos de información. En este sentido, entre 1999 a 2004, McGuiness (2005, 2006) realizó un estudio exploratorio de ámbito cualitativo con el objetivo de determinar la alfabetización informacional en el entorno de la formación graduada en la República de Irlanda; Partiendo de la convicción de que la colaboración entre bibliotecarios y profesores es uno de los elementos críticos en los programas de alfabetización informacional destinados a los estudiantes de grado, el objetivo era identificar los factores internos y externos existentes en el entorno académico y que constituyen barreras a esa colaboración. La autora partió de la idea defendida por Hardesty (1995) y Badke (2005) de que las creencias, percepciones y prácticas de trabajo que caracterizan a los profesores los impiden desarrollar actividades en colaboración con elementos externos, incluyendo a los bibliotecarios.

El focus de este estudio fueron profesores de Sociología y de Ingeniería Civil. Como técnica de recogida de datos se utilizó la entrevista semi-estructurada. Los participantes (20 profesores de cada área científica y 9 bibliotecarios) se seleccionaron en base a purposive sampling, es decir, porque estaban interesados en participar en el estudio.

La conclusión fue que la colaboración entre ambos grupos, en los términos definidos en la literatura, no existe, y los bibliotecarios son considerados por los profesores como meros suministradores de servicios. La mayoría de las interacciones entre los dos grupos tiene lugar en los recursos físicos que los profesores necesitan, sea para la investigación o para la docencia. Por otro lado, el 90\% de los profesores declaran que entienden que los bibliotecarios desarrollan o deben desarrollar un papel en la enseñanza, pero no les preocupa el asunto y ninguno de ellos los involucra directamente en sus cursos y "...no hay pruebas de que los dos grupos colaboren en otra cosa que alguna sesión ocasional en el aula o una vuelta por la biblioteca.» (p. 250).

Para muchos de ellos, la entrevista realizada en la investigación representó la primera vez que habían oído hablar de este tema. Entienden que la participación de los bibliotecarios en sus clases es difícil, sobre todo, por problemas de tiempo. Para otros, los bibliotecarios no poseen un conocimiento especializado en el 
tema que les permita participar en las actividades de docencia porque «mientras que los bibliotecarios son percibidos como expertos en su propia área, la biblioteconomía, los profesores no consideran que ello los cualifique para participar en la enseñanza de disciplinas diferentes a la suya.» (p. 250).

En cuanto al tipo de formación que los bibliotecarios podrían garantizar, ésta se dirige a la tradicional formación de usuarios (bibliographic instruction), es decir, la presentación de los recursos físicos y electrónicos de información de la biblioteca, la navegación por Internet y otros recursos en línea y, sobre todo, encontrar o localizar información. Además, los profesores entienden que «...los estudiantes de alguna manera absorben y desarrollan los conocimientos y habilidades necesarios a través del mismo proceso por el que preparan una parte de trabajos escritos y mediante la aplicación de las recomendaciones impuestas por sus supervisores.» (p. 577).

Los profesores encuestados sobre quién tiene la responsabilidad principal de que los estudiantes sean alfabetizados en información, a pesar de estar de acuerdo en que los bibliotecarios desarrollan un tipo de actividades de docencia muy específico, entienden que son ellos o los departamentos los que tienen el control sobre este proceso. En consecuencia «La alfabetización informacional se percibe simplemente como uno más de los imperativos del staff académico, y las respuestas de los participantes apuntaban a una tendencia a suponer que los estudiantes podrían «adquirirla» en el transcurso de sus estudios...» (p. 251).

En síntesis, sobre los factores internos y externos existentes en el entorno académico y que constituyen barreras a esa colaboración el primero es que los profesores afirman que los estudiantes desarrollan las competencias de alfabetización informacional de modo gradual e intuitivo (learning by doing), por tanto, es un proceso solitario en la medida que es el propio interés personal del estudiante el que determina su participación. El estudio ha permitido identificar que los profesores desconocen las cuestiones pedagógicas planteadas por el desarrollo de la alfabetización informacional.

En el Reino Unido la sensibilización por estos asuntos fue en aumento y ello supuso que en el año 2006, el Research Information Network (RIN) y el Consortium of Research Libraries (CURL) (2007) patrocinaran un estudio con el objetivo de conocer cómo los investigadores interactúan y perciben los servicios de las bibliotecas universitarias. Se realizó con 2.250 investigadores (con una tasa de respuesta del 2,25\%) y 300 bibliotecas y la información se recogió a partir de un cuestionario y de reuniones de grupos de discusión.

En abril de 2007 se publicó el Informe del proyecto. En él se comienza por destacar el importante papel desarrollado por las bibliotecas universitarias en el apoyo a la investigación señalando que la última década "...ha traído consigo un cambio radical en la relación entre los investigadores y las bibliotecas. Los avances tecnológicos y la disponibilidad de recursos de información en línea han cambiado la manera de llevar a cabo la investigación así como los servicios que las bibliotecas académicas proporcionan a sus comunidades investigadoras.» (p. 2). 
En este Informe se afirma que los investigadores consideran que las bibliotecas de sus instituciones están realizando de forma eficaz su tarea de recuperación y entrega de la información que ellos necesitan. También se afirma que los retos futuros son importantes y es necesario considerar nuevas funciones y responsabilidades de todos los implicados en el ciclo de investigación (investigadores, instituciones de investigación y organismos nacionales, así como bibliotecas).

Asimismo, se constatan algunos puntos de presión: los investigadores piensan que la financiación de la biblioteca debe ser una prioridad para la institución, los bibliotecarios indican que no siempre es fácil obtener su apoyo para presionar la gestión de los órganos de gobierno. Así, la escasez de los recursos obliga a una competición entre solicitantes "...muchos investigadores consideran que las bibliotecas dan mayor prioridad al apoyo a la enseñanza y al aprendizaje que a la investigación...» (p. 2).

Se comprueba la disminución en el número de visitas a la biblioteca en los últimos cinco años a consecuencia de la preferencia de los investigadores por el acceso a los recursos de información electrónicos a partir de su ordenador en su despacho o en su casa. Esto implica que los bibliotecarios tendrán que trabajar por aumentar los contenidos de información disponibles electrónicamente y también por cambiar la forma de suministrar los servicios de las bibliotecas.

Paralelamente a una disminución en las visitas a la biblioteca por parte de los investigadores, los estudiantes continúan utilizándola como un lugar para estudiar pero, ahora y cada vez más, en grupo, lo que significa que la propia configuración del espacio físico de la biblioteca, debe sufrir cambios.

Con relación al comportamiento de los investigadores en las búsquedas de información, continúan utilizándose los catálogos electrónicos. En este sentido, se atribuye gran importancia a la calidad de los metadatos.

Se verifica que los investigadores adoptan una perspectiva pragmática en la búsqueda de información y que «El préstamo interbibliotecario formal seguirá disminuyendo a medio plazo y los investigadores harán un uso cada vez mayor de mecanismos informales para compartir información.» (p. 37).

Igualmente, se afirma que el aumento del número de proyectos de investigación en colaboración y multidisciplinares plantean desafíos a las bibliotecas en cuanto a la oferta de servicios de información eficaces y equitativos, destacándose la necesidad de clarificar las funciones y responsabilidades de todos en la gestión del creciente volumen de resultados de la investigación digitales.

Las opiniones de los bibliotecarios y de los investigadores coinciden en cuanto al papel clave de los primeros en el futuro en lo que se refiere a la gestión de recursos digitales de información. Sin embargo, en cuanto a su papel en la formación en alfabetización informacional que continúa siendo fundamental por los bibliotecarios «...mientras que muchos investigadores están de acuerdo con ellos, existen problemas para que los bibliotecarios consigan una importante aceptación de su asesoramiento y experiencia.» (p. 56).

Respecto al papel futuro de las bibliotecas en el apoyo a la investigación siguen existiendo opiniones diferentes, lo que implica la necesidad de diálogo 
entre ellos para garantizar que los servicios se desarrollen y ofrezcan de la forma más eficaz.

La comunicación de los bibliotecarios con los investigadores está marcada por algunos problemas, de los cuales destaca «... la fugacidad de muchas de las relaciones individuales que se forman, la tendencia creciente de los investigadores a utilizar los servicios de biblioteca de forma remota así como su independencia.» (p. 3).

Por último, se considera que las bibliotecas necesitan proclamar su potencial para que los investigadores comprendan y reconozcan la importancia de la contribución a su trabajo. En ese sentido, "el éxito futuro de la investigación en las bibliotecas necesita forjar una identidad de marca más fuerte dentro de la institución» (p. 4).

La realización de todos estos estudios ha contribuido a clarificar ciertas percepciones que pueden ayudar a corregir malentendidos, a fomentar el diálogo y a mejorar las relaciones entre estos dos grupos llamados a entenderse, puesto que ambos tienen como preocupación principal a los estudiantes. Es exactamente en torno a estos últimos donde debe desarrollarse un conjunto de actividades que promuevan la colaboración entre bibliotecarios y profesores para que los primeros se transformen en «... verdaderos socios y compañeros educadores junto con el profesorado.» (Rapple, 1997b).

\section{Conclusiones}

De la revisión de estudios empíricos sobre las cuestiones abordadas en este trabajo se pueden extraer una serie de conclusiones, siempre con la precaución a la que nos obliga esta metodología de análisis, puesto que pueden darse casos concretos en los que no puedan aplicarse, dadas las particularidades tanto temporales como físicas, de los diferentes estudios de caso. No obstante, analizando las propias conclusiones de las investigaciones, podemos añadir:

En líneas generales, y a lo largo de las diferentes décadas analizadas, se mantiene la constante de que los profesores están satisfechos con la labor de la biblioteca y bibliotecarios de sus respectivas instituciones, aunque restringiendo su labor a la de servicio y apoyo. Así mismo, se hace patente la necesidad de que la biblioteca/bibliotecario realicen una labor de promoción de sus actividades que acabe con la «invisibilidad" de sus funciones.

No obstante, a través del tiempo se vislumbran cambios en las actitudes del profesorado respecto a los bibliotecarios y viceversa. Así, en la década de los 80 el interés por estos estudios empieza a ser evidente y, aunque persiste la visión del bibliotecario como personal de apoyo, empieza a surgir debate sobre su estatus. También se le reconoce como investigador pero únicamente en al área de la biblioteconomía. El aula sigue siendo territorio vetado para el bibliotecario respecto a asumir tareas docentes.

Por parte de los bibliotecarios se hace patente el problema de su propia identidad profesional y crece el interés por las percepciones que de ellos tiene el 
profesorado. La metodología más empleada para la realización de estos estudios se centra en análisis cuantitativos, sobre todo la encuesta.

En la década de los noventa los intereses de estos estudios versan sobre la necesidad de la existencia de contactos entre ambos colectivos, puesto que ello influye directamente en la percepción que los profesores tienen de su biblioteca, y en aconsejar a sus alumnos estar inmersos en la "cultura bibliotecaria». Los bibliotecarios empiezan a ser conscientes de la necesidad de hacerse visibles y recurrir incluso a técnicas de "marketing" para lograr sus objetivos. La metodología de trabajo empleada va a compaginar los estudios cuantitativos con los cualitativos a través de los "grupos de discusión" por considerarse más adecuados que las encuestas. Las ciencias sociales comienzan a interesarse por estos temas.

A partir del año 2000 la explosión de los recursos informativos electrónicos y la generalización del uso de internet, causan un giro muy importante en estos asuntos. La autosuficiencia de los usuarios en cuanto a la adquisición de información plantea nuevos retos a los bibliotecarios que insisten en la desconexión de ambos grupos y en la necesidad de poner en práctica la tarea de alfabetización informacional, no siempre bien entendida por los profesores.

Todo ello nos hace considerar que el colectivo de profesionales de bibliotecas universitarias debe consensuar sus acciones para acabar con una visión estereotipada sobre las mismas. Para ello, ha de asumir un papel «más agresivo», dinámico y activo en la misión educativa.

Los responsables de las bibliotecas universitarias necesitan adaptarse a los cambios del nuevo proceso enseñanza-aprendizaje incorporando nuevos valores a los ya tradicionales y definiendo nuevas prioridades, competencias y roles más dinámicos en la misión educativa de las instituciones superiores. Estas actuaciones deben enmarcarse dentro de la visión, misión y objetivos de la propia universidad, y en gran medida de los profesores que deben apoyar la colaboración de otros colectivos en la tarea docente. El gran desafío es el de continuar mejorando los servicios actuales, facilitar el acceso a recursos para que el profesorado pueda gestionar otras formas de enseñanza.

Es imprescindible establecer una política de comunicación entre los profesores y los bibliotecarios que refuerce la labor de ambos de cara a una mejora en los procesos de docencia-aprendizaje de los alumnos.

\section{Bibliografía}

Badke, W. (2005). Can't get no respect: helping faculty to understand the educational power of information literacy. The Reference Librarian, $\mathrm{n}^{\circ}$ 89-90, 63-80.

Breivik, P. (1989). Politics for closing the gap. The Reference Librarian. vol. 10 (24), 5-16.

Budd, J.; Coutant, P. (1981). Faculty perceptions of librarians: a survey. (Bethesda, Md.: ERIC Document Reproduction Service, ED 215 697, 1981) p. 31. 
Byron, S. (1997). Faculty perceptions of library support for teaching and research: a focus group study with selected faculty from the College of Arts and Sciences. Bethesda, Md.; ERIC Document Reproduction Service, ED 420 310, 1997, p. 11.

Carpenter, K. E. (1997). The Librarian-Scholar. The Journal of Academic Librarianship, vol. 23 (5), 398-401.

Christiansen, L.; Stombler, M.; Thaxton, L. (2004). A report on librarian-faculty relations from a sociological perspective. The Journal of Academic Librarianship, vol. 30 (2), 116-121.

Chu, F. (1997). Librarian-faculty relations in collection development. The Journal of Academic Librarianship, vol. 23 (1), 15-20.

Church, G. M. (2002). In the eye of the beholder: how librarians have been viewed over time. En: Arant, W.; Benefiel, C. R. (eds.) The image and role of the librarian. Haworth Information Press, Binghamton, NY, EE. UU

Cook, M. K. (1981). Rank, status, and contribution of academic librarians as perceived by the teaching faculty at Southern Illinois University, Carbondale. College \& Research Libraries, vol. 42 (3), 214-223.

Davis, J. Y.; Bentley, S. (1979). Factors affecting faculty perceptions of academic libraries. College E Research Libraries, vol. 40 (6), 527-532.

Dilmore, D. H. (1996). Librarian/Faculty interaction at nine New England colleges. College \& Research Libraries, vol. 57, 274-284.

Divay, G.; Ducas, A. M.; Michaud-Oystryck, N. (1987). Faculty perceptions of librarians at the University of Manitoba. College $E$ Research Libraries, vol. 48 (1), 28-35.

Ducas, A. M.; Michaud-Oystryk, N. (2003). Toward a new enterprise: capitalizing on the faculty-librarian partnership. College \& Research Libraries, vol. 64 (1), 55-74.

Farber, E. (1999a). Faculty-librarian cooperation: a personal retrospective. Reference Services Review, vol. 27 (3), 229-234.

Farber, E. (1999b). College libraries and the teaching/learning process: a 25-year reflection. The Journal of Academic Librarianship, vol. 25 (3), 171-177.

Feldman, D.; Sciammarella, S. (2000). Both sides of the looking glass: librarian and teaching faculty perceptions of librarianship at six community colleges. College $\&$ Research Libraries, vol. 61 (6), 491-498.

Hardesty, L. (1995). Faculty culture and bibliographic instruction: an exploratory analysis. Library Trends vol. 44 (2), 339-367. Disponible en: <http://hdl.handle.net/2142/8028> [consultado el 31 de octubre 2008].

Hardesty, L. (1999). Reflections on 25 years of library instruction: have we made progress? Reference Services Review, vol. 27 (3), 242-246.

Hawkins, B.; Battin, P. (1997). The changing role of the information resources professional: a dialogue. CAUSE/EFFECT, vol. 20 (1), 22-30. Disponible en Internet: <http://www. cause.org/information-resources/ir-library/html/cem9717.html> [consultado el 21 de diciembre de 2007].

Hutchins, E. O. (2005). Building strong collaborative relationships with disciplinary faculty. En: Gregory, G. M. (editor). The successful academic librarian: winning strategies from library leaders. Information Today, Inc. New Jersey, NJ, EE. UU.

Iannuzzi, P. (1998). Faculty development and information literacy: establishing campus partnerships. Reference Services Review, vol. 26, 97-116. 
Ivey, R. T. (1994). Research notes: teaching faculty perceptions of academic librarians at Memphis State University. College \& Research Libraries, vol. 55 (1), 69-82.

Jenkins, P. (2005). Faculty-librarian relationships. Oxford (England); Chandos Publishing Limited, p. 166.

Jordan, P. (1998). The academic library and its users. Aldershot (England), Brookfield (USA); Gower, p. 168.

Kotter, W. R. (1999). Bridging the great divide: improving relations between librarians and classroom faculty. The Journal of Academic Librarianship, vol. 25 (4), 294-303.

Manuel, K.; Molloy, M.; Beck, S. (2003). What faculty wants: a study of attitudes influencing faculty collaboration in library instruction. Proceeding ACRL Eleventh National Conference, p. 21-26. [en línea]. Charlotte, NC, EE. UU: ACRL. Disponible en Internet: http://www.ala.org/ala/acrl/acrlevents/manuel/pdf [consultado el 21 de noviembre de 2007].

Marchant, M. P. (1969). Faculty-librarian conflict. Library Journal, n 94, 2886-2889.

McGuiness, C. (2005). Attitudes of academics to the library's role in information literacy education. En: Martin, A.; Rader, H. (editores) Information and IT literacy: enabling learning in the 21st century. Facet Publishing, Londres.

McGuiness, C. (2006). What faculty think - exploring the barriers to information literacy development in undergraduate education? The Journal of Academic Librarianship, vol. 32 (6), 57-582.

Oberg, L. R.; Schleiter, M. K.; Van Houten, M. (1989). Faculty perceptions of librarians at Albion College: status, role, contribution, and contacts. College \& Research Libraries, vol. 50 (2), 215-230.

Peacock, J. (2001). Teaching skills for teaching librarians: postcards from the edge of the educational paradigm. Australian Academic \& Research Libraries, vol. 32 (1), 1-11 Disponible en Internet: <http://www.accessmylibrary.com/coms2/summary_0286-10728272_ITM> [consultado el 15 de enero 2007].

Rader, H. (2004). Building faculty-librarian partnerships for information fluency: the time for sharing information expertise is now. College \& Research Libraries News, vol. 65, 74-76.

Rapple, B. A. (1997b). The librarian as teacher in the networked environment. College Teaching vol. 45 (3), 114-116. Disponible en Internet: <http://web.ebscohost.com/ ehost/detail?vid=3\&hid=16\&sid=d3465b07-93ec-48ba-ac56-318fca6d9fdb\%40SRCSMZ> [consultado el 27 de febrero de 2008].

Raspa, D.; Ward, D. (2000). Introduction. En: Raspa, D.; Ward, D. (editores). The collaborative imperative: librarians and faculty working together in the information universe. Association of College and Research Libraries, Chicago, IL, EE. UU.

Research Information Network (RIN) y Consortium of Research Libraries (CURL) (2007). Researchers' use of academic libraries and their services: a report commissioned by the Research Information Network and the Consortium of Research Libraries [en línea]. Disponible en Internet: http://www.rin.ac.uk/files/libraries-report-2007.pdf [consultado el 12 de noviembre de 2007].

Winner, M. (1998). Librarians as partners in the classroom: an increasing imperative. Reference Services Review, vol. 26, 25-29. 